\title{
First Cases of Natural Infections with Borrelia hispanica in Two Dogs and a Cat from Europe
}

\author{
Gabriele Margos ${ }^{1, *, \dagger}$, Nikola Pantchev ${ }^{2, \dagger}$, Majda Globokar ${ }^{2}$, Javier Lopez ${ }^{3}$, Jaume Rodon ${ }^{3}$, \\ Leticia Hernandez $^{3}$, Heike Herold ${ }^{4} \mathbb{D}$, Noelia Salas ${ }^{3}$, Anna Civit ${ }^{3}$ and Volker Fingerle ${ }^{1}$ \\ 1 German National Reference Centre for Borrelia, Bavarian Health and Food Safety Authority, \\ 85764 Oberschleißheim, Germany; volker.fingerle@lgl.bayern.de \\ 2 IDEXX Laboratories, 70806 Kornwestheim, Germany; nikola-pantchev@idexx.com (N.P.); \\ majda-globokar@idexx.com (M.G.) \\ 3 IDEXX Laboratories, 08038 Barcelona, Spain; javier-lopez@idexx.com (J.L.); jaume-rodon@idexx.com (J.R.); \\ leticia-hernandez@idexx.com (L.H.); noelia-salas@idexx.com (N.S.); anna-civit@idexx.com (A.C.) \\ 4 Bavarian Health and Food Safety Authority, 85764 Oberschleißheim, Germany; herold.heike@web.de \\ * Correspondence: Gabriele.Margos@lgl.bayern.de \\ + These authors contributed equally to this work.
}

Received: 21 July 2020; Accepted: 14 August 2020; Published: 18 August 2020

\begin{abstract}
Canine cases of relapsing fever (RF) borreliosis have been described in Israel and the USA, where two RF species, Borrelia turicatae and Borrelia hermsii, can cause similar clinical signs to the Borrelia persica in dogs and cats reported from Israel, including fever, lethargy, anorexia, thrombocytopenia, and spirochetemia. In this report, we describe the first clinical cases of two dogs and a cat from Spain (Cordoba, Valencia, and Seville) caused by the RF species Borrelia hispanica. Spirochetes were present in the blood smears of all three animals, and clinical signs included lethargy, pale mucosa, anorexia, cachexia, or mild abdominal respiration. Laboratory findings, like thrombocytopenia in both dogs, may have been caused by co-infecting pathogens (i.e., Babesia vogeli, confirmed in one $\mathrm{dog}$ ). Anemia was noticed in one of the dogs and in the cat. Borrelia hispanica was confirmed as an infecting agent by molecular analysis of the 16S rRNA locus. Molecular analysis of housekeeping genes and phylogenetic analyses, as well as successful in vitro culture of the feline isolate confirmed the causative agent as B. hispanica.
\end{abstract}

Keywords: Borrelia hispanica; Europe; relapsing fever; borreliosis; cat; dog

\section{Background}

The genus Borrelia comprises three phylogenetic clusters, namely: Lyme Borreliosis (LB) borreliae, relapsing fever $(\mathrm{RF})$ borreliae, and the recently described reptile-associated and echidna-associated Borrelia (REP borreliae, Borrelia (B.) turcica, and Candidatus B. tachyglossi, respectively) [1,2].

LB in dogs in Europe has been described as a condition of arthritis and/or glomerulonephritis; the incidence of clinical disease is rare, despite a high exposure of dogs to the tick vector (Ixodes ricinus), and there may be a breed/genetic predisposition (reviewed by the authors of [3]). LB in cats in Europe is a rare condition - the seroprevalence is significantly lower compared with dogs, which may, in part, be explained by different tick exposures [4].

Apart from LB species, which are vectored by hard ticks of the genus Ixodes, soft ticks in Europe may carry Borrelia species that belong to the RF group of spirochetes, in particular Borrelia hispanica. Borrelia hispanica represents a spirochete species transmitted by Ornithodoros ticks, which can cause TBRF (tick-borne relapsing fever) in humans in the Mediterranean area [5,6]. Trape et al. [7] detected B. hispanica in O. marocanus, O. occidentalis, and O. kairouanensis. They represent a complex of three 
large species of soft ticks (Argasidae) of the genus Ornithodoros (female adult length 5.5-7.5 mm), and are distributed in characteristic Mediterranean regions in Morocco, Algeria, Tunisia, and Spain. These species were previously confused with O. erraticus [7]. Up until now, however, no cases of B. hispanica in dogs and cats have been reported in Europe.

Cases of RF borreliosis have been reported in dogs and cats from Israel naturally infected with B. persica, a Borrelia species that is transmitted by O. tholozani in the Middle East, Egypt, Central Asia, and India. The main clinical findings in cats included lethargy, anorexia, anemia, and thrombocytopenia, while fever $\left(>39^{\circ} \mathrm{C}\right)$ was reported in only one out of five cats. Dogs were lethargic, anorexic, anemic, and showed fever; the majority were also thrombocytopenic. Three dogs out of five were co-infected with B. persica and Babesia. The animals were all treated with antibiotics (tetracyclines, penicillins, fluorochinolons, or combinations of them), the dogs co-infected with Babesia received additional imidocarb diproprionate, and the survival rate of both dogs and cats was $80 \%$ [8].

Furthermore, canine cases of RF borrelia are described from the U.S., where two RF species, B. turicatae and B. hermsii, transmitted by Ornithodoros ticks, can cause similar clinical signs as B. persica in dogs and cats, including fever, lethargy, anorexia, thrombocytopenia, and spirochetemia. Neurologic disease symptoms have also been described [9].

In this report, we describe the first clinical cases of two dogs and a cat caused by B. hispanica in Europe, with spirochetes present in blood smears, molecular analysis, and successful in vitro cultivation of one isolate.

\section{Case Reports}

\subsection{Canine Case 1 (Dog 1; Sample ID VM531519)}

Anamnesis: Podenco (hound); short hair; male; 6 years old; approximately $10 \mathrm{~kg}$ body weight (BW); hunting dog living in La Luisiana, Seville (Spain); and sleeps under a roof. The following prophylactic measures were established: imidacloprid, chlorfenvinphos, and diazinon; no prophylaxis for leishmaniosis; treatment for internal parasites using albendazole and a combination of praziquantel/pyrantel/febantel; and annual rabies vaccine plan.

The dog was presented to a local veterinarian in February (3 February 2014) being lethargic, with pale mucosa, anemia (hematocrit $30.2 \%$ ), and thrombocytopenia (Table 1). No data about the occurrence of microorganisms in a stained blood smear were available at this time point. Because of a positive Ehrlichia canis serologic result ( $\left.\mathrm{SNAP}^{\circledR} 4 \mathrm{Dx}^{\circledR}\right)$ ), treatment was performed with $5 \mathrm{mg} / \mathrm{kg} / \mathrm{BW}$ of doxycycline, every $12 \mathrm{~h}$, for three weeks. Despite a good appetite, at the end of the first doxycycline cycle (27 February 2014), the hematocrit was still low (20.9\%). In March (4 and 13 March 2014), the hematocrit was still not within the reference range (38.3-56.5\%), with values of $32.8 \%$ and $31.4 \%$, respectively. Therefore, a second course of doxycycline, as described above, was performed. The dog improved clinically and by the end of March 2014 showed no more anemia. On 30 October 2014, the dog showed non-regenerative anemia (hematocrit $29.4 \%$, erythrocytes $3.98 \mathrm{~m} / \mu \mathrm{L}$, hemoglobin $8.9 \mathrm{~g} / \mathrm{dL}$, and reticulocytes $58,108 / \mu \mathrm{L}$ ), thrombocytopenia ( $23 \mathrm{tsd}$.), mild lymphopenia, and eosinopenia, as well as many spirochetes in a Giemsa-stained blood smear (Figure 1A, left panel). A diagnosis of relapsing fever was established and treatment with doxycycline was started the following day at the dose and interval mentioned above. Unfortunately, no information is available about the efficacy of the treatment and clinical progress. 
Table 1. Clinical signs and clinicopathological abnormalities in Borrelia hispanica infected animals.

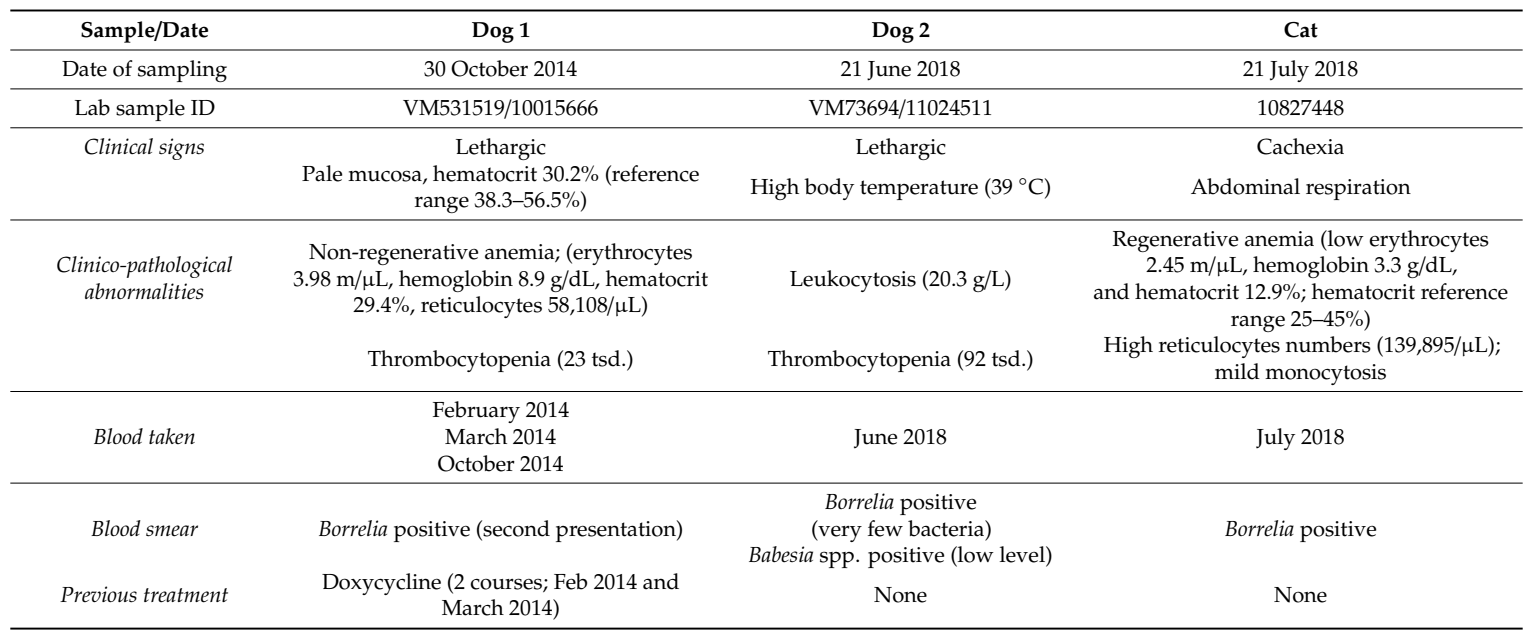

A
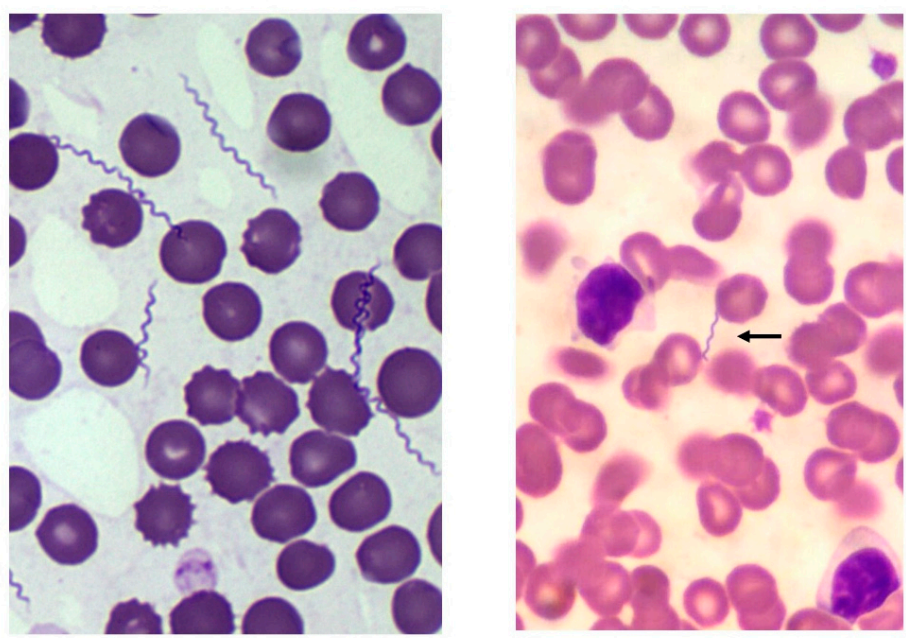

B

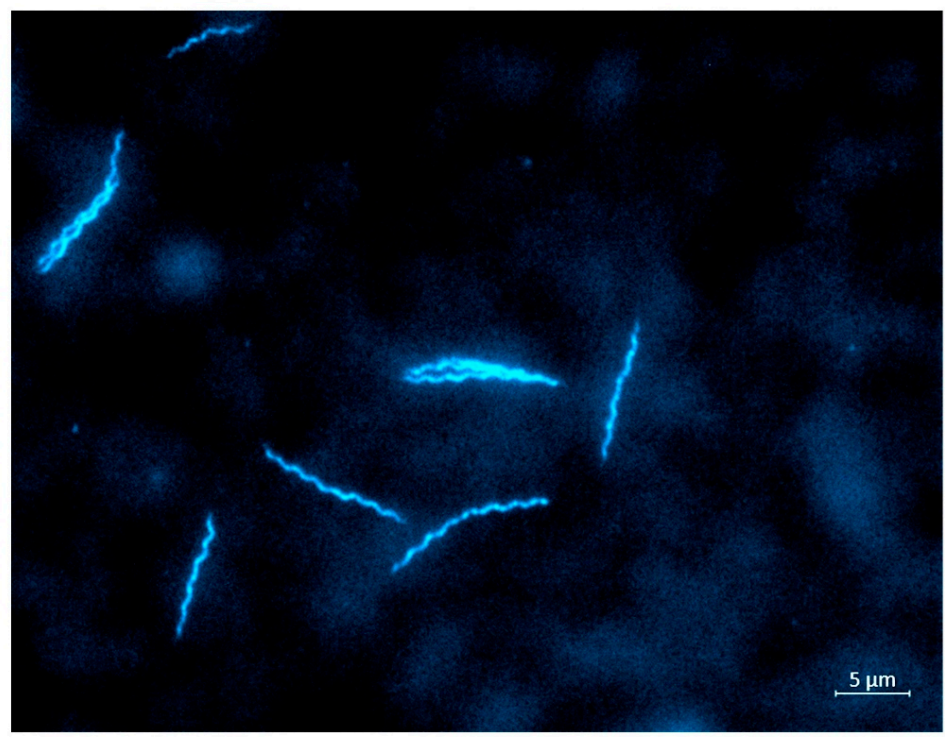

Figure 1. Spirochetes in Giemsa-stained blood smears of dog 1 (A, left panel, magnification 150×), dog 2 (A, right panel, magnification 100×, arrow pointing to Borrelia), and 4',6-diamidino-2-phenylindole (DAPI) stained Borrelia from an in vitro culture of infected cat blood (B). 
The laboratory tests were performed at IDEXX Ludwigsburg, Germany (November 2014).

PCR: At IDEXX Ludwigsburg, a diagnostic real-time PCR was conducted for B. burgdorferi sensu lato (target gene was flagellin $\mathrm{B}(\mathrm{flaB})$ ) with a negative result.

Serology: the dog showed $66 \mathrm{U} / \mathrm{mL}$ in the Quant-C6-Enzyme-linked immunosorbent assay (ELISA) (IDEXX; cut off $10 \mathrm{U} / \mathrm{mL}$ ), $25 \mathrm{VE}$ (Virotech units) measured by a whole-cell based IgG ELISA (Virotech; without VlsE; cut off $12 \mathrm{VE}$ ). The $\mathrm{SNAP}^{\circledR} 4 \mathrm{Dx}{ }^{\circledR}$ (IDEXX) test was negative, apart from a positive reaction against p30/p30-1 peptides of Ehrlichia canis. The Borrelia immunoblot (Viramed) showed two positive bands (p14 and VlsE), and four weakly positive bands (DbpA (Osp17), p21, OspC, and p39; data not shown). The sample showed a very high E. canis antibody titre in the immunofluorescence assay (IFA; MegaCor; >1:2560 cut off 1:40), a low Leishmania antibody level in ELISA (Afosa; 15.8 test units (TU); positive cut off 12 TU), and also a low positive Babesia antibody level of 23.5 TU (Afosa; positive cut off $19 \mathrm{TU})$.

\subsection{Canine Case 2 (Dog 2; Sample ID VM736940)}

Anamnesis: This dog lives in the Gandia area (Valencia), in a residential area in the countryside. It is an outdoor dog and sleeps in the courtyard. It was usually dewormed with a combination of febantel/pyrantel/praziquantel, and treated for ectoparasites with a deltamethrin-containing collar on an irregular basis. The dog showed anorexia and apathy at a body temperature of $39^{\circ} \mathrm{C}$ (Table 1 ). Within a blood smear, low level of Babesia spp. was observed and only few Borrelia were visible (Figure 1A, right panel); the dog showed no anemia, but only leukocytosis $(20.3 \mathrm{~g} / \mathrm{L})$ based on lymphocytosis $(11,182 / \mu \mathrm{L})$ and monocytosis $(1130 / \mu \mathrm{L})$, as well as thrombocytopenia (92 tsd.). Further blood values (including clinical chemistry) were within the normal range.

Serology (IDEXX Ludwigsburg): The SNAP ${ }^{\circledR} 4 \mathrm{Dx}^{\circledR}$ (IDEXX) test as well as two Borrelia ELISA tests, Quant-C6-ELISA (IDEXX) and the whole-cell based IgG ELISA (Virotech ${ }^{\circledR}$ ), were negative (no immunoblot was performed).

PCR (IDEXX Ludwigsburg): Real-time PCRs performed for Leishmania spp. (GP63), Ehrlichia spp. (dsb), Leptospira spp. (lipl32/hap-1), B. burgdorferi sensu lato (flaB), Anaplasma platys (groEL), and Anaplasma phagocytophilum (msp2) were negative; Babesia vogeli DNA (hsp 70) was amplified.

Treatment with doxycycline (for four weeks) and amoxicillin (for two weeks) was started after the suspected diagnosis of a spirochetal infection. After treatment, the dog recovered completely.

\subsection{Feline Case (Cat; Sample ID 10827448)}

Anamnesis: one-year old street cat, male, plenty of fleas, and no tick prophylaxis.

At presentation, the cat showed cachexia (extreme weight loss), mild abdominal respiration, and had a good appetite (Table 1). The body temperature was not elevated at the time of Borrelia detection. The cat was living in the Cordoba area (Andalucia). Laboratory abnormalities included severe regenerative anemia (low erythrocytes $(2.45 \mathrm{~m} / \mu \mathrm{L})$, hemoglobin $(3.3 \mathrm{~g} / \mathrm{dL})$, and hematocrit $(12.9 \%)$ at high reticulocyte numbers $(139,895 / \mu \mathrm{L}))$ and mild monocytosis. No other clinic-pathological abnormalities were found.

Serology (IDEXX Ludwigsburg): FeLV (Feline leukemia virus antigen)/FIV (feline immunodeficiency virus antibodies) (Uranotest ${ }^{\circledR}$ ) and SNAP ${ }^{\circledR} 4 D x^{\circledR}$ (IDEXX) tests were performed and came back negative; spirochetes were clearly visible in a Giemsa-stained blood smear (not shown).

Treatment was started with doxycycline after an additional blood sample was drawn for culture and molecular analyses. The duration of antibiotic treatment was 30 days. No clinical signs were observed after the treatment, and all of the altered values of hematology returned to normal. 


\section{Methods}

\subsection{Samples Included in Our Study}

All of the samples originated from animals living in Spain. The samples were sent to the IDEXX laboratory in Barcelona from local veterinarians, where the animals were presented because of clinical signs. The samples were obtained as part of a routine diagnostic evaluation. Written informed consent was obtained from the owner. All investigations comply with the current laws of the countries in which they were performed. Diagnostic laboratory analyses (serology and PCR) were performed at the IDEXX reference laboratories in Ludwigsburg/Germany, and were subsequently sent for culture and further molecular analyses to the German National Reference Center for Borrelia at the Bavarian Health and Food Safety Authority, Oberschleißheim/Germany. The sample IDs are as follows: dog $1=$ VM531519; cat $=10827448 ; \operatorname{dog} 2=$ VM736940.

\subsection{Serology IDEXX Ludwigsburg, Germany}

All of the tests were performed according to the literature $[3,10,11]$. For the immunoblot, slight modifications were implemented. Briefly, the Borrelia + OspA/B ViraStripe IgG Testkit (Viramed; Planegg, Germany) was performed, which contains specific purified antigens from B. afzelii (PKo) and B. burgdorferi sensu stricto, as well as a recombinant VlsE. As a secondary antibody, a phosphatase-labeled affinity purified antibody to dog $\operatorname{IgG}(\mathrm{H}+\mathrm{L}$; produced in goat) at a dilution of 1:500 was used.

\subsection{PCRs IDEXX Ludwigsburg, Germany}

All of the tests were performed according to the literature $[10,11]$, apart from Borrelia and Leptospira. Briefly, the total nucleic acid was extracted from the blood by using a QIAamp DNA Blood Mini kit (QIAGEN; Hilden, Germany), according to the manufacturer's instructions. A real-time-PCR assay was performed using the LightCycler 480 (Roche, Mannheim, Germany) with proprietary forward, reverse primers, and hydrolysis probes. The target gene for Leptospira spp. was the lipl32/hap-1 (accession number AF245281.1) and for B. burgdorferi sensu lato flaB (MF150071.1). The PCR assays that were employed during this study were shown to have a reproducible average analytical sensitivity of 10 DNA molecules per reaction.

\subsection{In Vitro Culture from Cat Blood at NRZ Borrelia, Oberschleißheim, Germany}

The Ethylenediaminetetraacetic acid (EDTA) blood obtained from the cat was used to set up in vitro cultures in microwell plates. All of the cultures were kept at $33{ }^{\circ} \mathrm{C}$ under a $5 \% \mathrm{CO}_{2}$ atmosphere. Four different media were used for the in vitro cultivation: (1) Modified-Kelly-Pettenkofer (MKP) medium (MKP basic medium supplemented with 5\% bovine serum albumin and 6\% rabbit serum) [12], (2) Barbour-Stoenner-Kelly (BSK)-H complete medium (Sigma-Aldrich; Darmstadt, Germany), (3) BSK-Y medium, and (4) RF-medium (MKP basic medium supplemented with bovine serum albumin (5\%) and 50\% fetal calf serum (FCS); CC-pro, Germany) [13]. For all of the cultures, $10 \mu \mathrm{L}$ of EDTA blood was placed into $300 \mu \mathrm{L}$ of medium.

\subsection{DNA Extraction and PCR (NRZ Borrelia, Oberschleißheim)}

DNA was extracted from the EDTA blood using the Maxwell ${ }^{\circledR} 16$ Blood DNA Purification Kit according to the manufacturer's instructions (Promega, Mannheim, Germany). The extracted DNA was subjected to PCR amplification, as described below.

Fragments of the 16S rRNA were amplified using primers and PCR conditions, as described previously [14]. Multilocus sequence typing (MLST) on housekeeping genes (clpA, clpX, nifS, pepX, pyrG, $r p l B, r e c G$, and $u v r A$ ) was performed principally, as described (see www.pubmlst.org/borrelia, [15]). The sequences for the primers used are given in Table 2. For all of the PCR reactions, HotStarTaq Mastermix (Qiagen, Germany) was used. A touch-down protocol was employed for the first nine 
cycles with annealing temperatures of $55^{\circ} \mathrm{C}$ to $48^{\circ} \mathrm{C}$, decreasing by $1{ }^{\circ} \mathrm{C}$ each cycle, followed by 32 cycles at a $48{ }^{\circ} \mathrm{C}$ annealing temperature. The temperature profile was $95^{\circ} \mathrm{C}$ for 15 min (activation of Taq polymerase), denaturation $94^{\circ} \mathrm{C}$ for $30 \mathrm{~s}$, annealing $30 \mathrm{~s}$, and elongation $72{ }^{\circ} \mathrm{C}$ for $60 \mathrm{~s}$. A final step of elongation was at $72{ }^{\circ} \mathrm{C}$ for $5 \mathrm{~min}$, and the samples were maintained at $12{ }^{\circ} \mathrm{C}$.

Table 2. Primer * used to amplify MLST housekeeping genes in Borrelia hispanica.

\begin{tabular}{|c|c|c|}
\hline Locus & Primer Name & Primer Sequence \\
\hline $\operatorname{clp} A$ & clpAF1258 & 5'-GATAAAGCTTTTGAYYTATTAGATGG-3 \\
\hline $\operatorname{clp} A$ & clpAR2276 & 5'-TCATATTTDATRGTDTCGTC-3' \\
\hline $\operatorname{clpX}$ & clpXF104 & 5'-CTGTTGCYATTTGTTTTGAATGYTC-3' \\
\hline $\operatorname{clpX}$ & clpXR1277 & 5'-TAAAGTTCTTTTGCCCAAGG-3' \\
\hline pepX & pepF361 & 5'-AGAGAYTTAAGYTTAKCAGG-3' \\
\hline pepX & pepR1207 & 5'-CYATAGTTTCTCTTAAAGAYTGC-3 \\
\hline pyrG & pyrF379 & 5'-TATTTAGGKAGAACTGTACAGC-3 \\
\hline pyrG & pyrR1375 & 5'-CAAGTCGCATTGTWGCAC-3 \\
\hline $\operatorname{rec} G$ & recF898 & 5'-GCКTTTCTMTCTAGYATTCC-3 \\
\hline recG & recR1779 & 5'-TTCRGTTAAAGGTTCCTTATAAAG-3 \\
\hline rplB & rplF3 & 5'-GGAGAAAAATATGGGKATTAAGAC-3 \\
\hline$r p l B$ & rplR769 & 5'-GRCCCCAAGGWGATAC-3 \\
\hline uvrA & uvrF1170 & 5'-GAGGCGTTATCTTWCAAC-3 \\
\hline uvrA & uvrR2181 & 5'-AGACTCTGGAAGCTTWGC-3 \\
\hline
\end{tabular}

\subsection{Molecular Analyses}

Commercial sequencing was done by GATC Biotech AG (Konstanz, Germany). We used MEGA5 [16,17] for sequence alignment, genetic distance analyses, and construction of phylogenetic trees. The Basic Local Alignment Search Tool (BLAST) [18] was used to compare the sequences obtained here to the sequences in GenBank using standard settings. Genetic distance analyses were conducted in MEGA5 [16,17] using the Kimura 2-parameter model [19]. The evolutionary history was inferred by using the maximum likelihood method based on the general time reversible model [16,20]. The initial tree(s) for the heuristic search were obtained automatically by applying neighbor-joining and BioNJ algorithms to a matrix of pairwise distances estimated using the maximum composite likelihood (MCL) approach, and then selecting the topology with a superior log likelihood value. The bootstrap values were calculated for 1000 repetitions. A discrete Gamma distribution was used to model the evolutionary rate differences among sites $(+G)$. The rate variation model allowed for some sites to be evolutionarily invariable $(+\mathrm{I})$. The trees are drawn to scale, with branch lengths measured in the number of substitutions per site. The codon positions included were 1 st +2 nd +3 rd+Noncoding for the housekeeping gene sequences. All of the positions containing gaps and missing data were eliminated. Further information is given in the figure legend.

\subsection{Sequence Deposition}

The 16S rRNA sequences were submitted to GenBank with accession numbers MN173954 (cat) and MN175320 (dog2). The sequences of the MLST housekeeping loci can be obtained from the Borrelia MLST database under pubmlst.org/borrelia/. Allele numbers are given in Table 3. 
Table 3. Allele numbers, GenBank accession numbers, and Basic Local Alignment Search Tool (BLAST) hits in GenBank.

\begin{tabular}{|c|c|c|c|c|}
\hline Locus/Sample & Species and Isolate & Coverage & Similarity & GenBank Accession Number \\
\hline \multirow{5}{*}{ clpX/dog1 (\#allele 210)/cat (\#allele 210) } & Borrelia crocidurae str. Achema & $100 \%$ & $97 \%$ & СР003426.1 \\
\hline & Borrelia recurrentis $\mathrm{A} 1$ & $100 \%$ & $97 \%$ & СР000993.1 \\
\hline & Borrelia duttonii Ly & $100 \%$ & $97 \%$ & СР000976.1 \\
\hline & Borrelia crocidurae DOU & $100 \%$ & $96 \%$ & СР004267.1 \\
\hline & Borrelia persica strain LMU-T01 & $99 \%$ & $91 \%$ & KP826804. \\
\hline \multirow{5}{*}{ pepX/cat (\#allele 263) } & Borrelia recurrentis $\mathrm{A} 1$ & $100 \%$ & $98 \%$ & СР000993.1 \\
\hline & Borrelia crocidurae DOU & $100 \%$ & $97 \%$ & СР004267.1 \\
\hline & Borrelia crocidurae str. Achema & $100 \%$ & $97 \%$ & СР003426.1 \\
\hline & Borrelia duttonii Ly & $99 \%$ & $97 \%$ & СР000976.1 \\
\hline & Borrelia persica strain LMU-T01 & $100 \%$ & $90 \%$ & KP826805.1 \\
\hline \multirow{4}{*}{ pyrG/dog1 (\#allele 274) } & Borrelia crocidurae str. Achema & $100 \%$ & $98 \%$ & СР003426.1 \\
\hline & Borrelia recurrentis $\mathrm{A} 1$ & $100 \%$ & $97 \%$ & СР000993.1 \\
\hline & Borrelia duttonii Ly & $100 \%$ & $97 \%$ & СР000976.1 \\
\hline & Borrelia persica strain LMU-T01 & $100 \%$ & $89 \%$ & KP826806.1 \\
\hline \multirow{5}{*}{ pyrG/cat (\#allele 273) } & Borrelia crocidurae DOU & $100 \%$ & $98 \%$ & СР004267.1 \\
\hline & Borrelia crocidurae str. Achema & $100 \%$ & $98 \%$ & СР003426.1 \\
\hline & Borrelia recurrentis A1 & $100 \%$ & $98 \%$ & СР000993.1 \\
\hline & Borrelia duttonii Ly & $100 \%$ & $98 \%$ & СР000976.1 \\
\hline & Borrelia persica strain LMU-T01 & $100 \%$ & $90 \%$ & KP826806.1 \\
\hline \multirow{5}{*}{ recG/cat (\#allele 288) } & Borrelia crocidurae str. Achema & $100 \%$ & $97 \%$ & СР003426.1 \\
\hline & Borrelia crocidurae DOU & $100 \%$ & $97 \%$ & СР004267.1 \\
\hline & Borrelia duttonii Ly & $100 \%$ & $97 \%$ & СР000976.1 \\
\hline & Borrelia recurrentis A1 & $100 \%$ & $96 \%$ & СР000993.1 \\
\hline & Borrelia persica strain LMU-T01 & $99 \%$ & $89 \%$ & KP826807.1 \\
\hline \multirow{5}{*}{ rplB/dog1 (\#allele 206) } & Borrelia crocidurae str. Achema & $100 \%$ & $98 \%$ & СР003426.1 \\
\hline & Borrelia recurrentis A1 & $100 \%$ & $98 \%$ & СР000993.1 \\
\hline & Borrelia duttonii Ly & $100 \%$ & $98 \%$ & СР000976.1 \\
\hline & Borrelia crocidurae DOU & $100 \%$ & $98 \%$ & СР004267.1 \\
\hline & Borrelia persica strain LMU-T01 & $98 \%$ & $92 \%$ & KP826807.1 \\
\hline \multirow{5}{*}{ 16S rRNA/dog2 (* MN175320)/cat (* MN173954) } & Borrelia hispanica strain CR1 & $100 \%$ & $100 \%$ & GU350705.1 \\
\hline & Borrelia hispanica 16S rRNA gene & $100 \%$ & $100 \%$ & DQ057988.1 \\
\hline & Borrelia crocidurae str. Achema & $100 \%$ & $99 \%$ & СР003426.1 \\
\hline & Borrelia hispanica strain Sp3 & $100 \%$ & $99 \%$ & GU350706.1 \\
\hline & Borrelia crocidurae strain $7-10 \mathrm{TO} 58$ & $100 \%$ & $99 \%$ & GQ358198.1 \\
\hline
\end{tabular}

\# allele numbers and isolate information are available at the Borrelia MLST database pubmlst.org/borrelia/; * GenBank accession number.

\section{Results and Discussion}

The report presented here describes the first clinical cases of natural infections with B. hispanica in two dogs and one cat living in various places in Spain (Cordoba, Valencia, and Seville). Clinical signs were reported as lethargy, pale mucosa, anorexia, cachexia, or mild abdominal respiration, and were consistent with previously described symptoms in animals infected with different RF Borrelia species $[8,9]$.

Dog 1 (sample ID VM531519) represents a case with clearly visible spirochetes in the blood (Figure 1A) that were identified as B. hispanica by molecular analysis. This dog had the following antibodies to other canine vector-borne diseases (CVBDs): high E. canis titer but also low Leishmania and Babesia antibodies. Thus, the clinical signs and laboratory abnormalities (such as non-regenerative anemia and thrombocytopenia, as well as a mild lymphopenia and eosinopenia) could have been triggered or exacerbated by the presence of a co-infection. Another explanation may be that the dog experienced an Ehrlichia-infection in early February 2014-which was successfully treated-and then acquired an RF Borrelia infection that was diagnosed in October 2014. Co-infections in animals with different disease agents should always be considered; in our study, dog 2 (sample ID VM736940) showed a co-infection of B. hispanica (microscopically/molecular) and Ba. vogeli (microscopically/molecular). In a previous study on dogs infected with B. persica and co-infected with Babesia (PCR and microscopy/blood smear), additional treatment with imidocarb was initiated [8], but this was not performed in the present case as $\operatorname{dog} 2$ improved with only antibiotic treatment (amoxicillin and doxycycline).

Interestingly, dog 1 showed a positive serologic reaction to B. burgdorferi in ELISA, whereas dog 2 did not. Possible reasons for these differences in the diagnostic response include an actual exposure to both (relapsing fever and Lyme borrelia) in $\operatorname{dog} 1$; an anamnestic titer against B. burgdorferi, after a previous successfully cleared infection; or a varied cross-reactivity of antibodies (i.e., IgM) against RF Borrelia with B. burgdorferi due to different time points of infection. Support for the latter supposition 
comes from the fact that dog 1 showed a high number of spirochetes in the blood smear (Figure 1A, left panel), whereas only a few bacteria were identified in the blood smear of dog 2 (Figure 1A, right panel). Moreover, dog 2 was the only animal without anemia, and showed signs pointing toward chronic infection, such as leucocytosis and thrombocytopenia. A recent experimental infection of six dogs with the RF species $B$. turicatae lends support to the cross-reactivity hypothesis, as five of the dogs showed positive results in a whole-cell-based test (IFA) for LB borreliae, three of them had slight positive reactions in a Quant-C6-ELISA (13 to $24 \mathrm{U} / \mathrm{mL}$ ), but all six dogs reacted negatively in the SNAP ${ }^{\circledR} 4 \mathrm{Dx}^{\circledR}$ test (as shown for both dogs and the cat in the present study) [21]. A limitation of this study was that only one time point post infection ( $43 \mathrm{dpi}$ ) was tested. Therefore, the SNAP ${ }^{\circledR} 4 \mathrm{Dx}{ }^{\circledR}$ test utilizing a C6 peptide of B. burgdorferi can currently be regarded as the only serological test for LB in dogs not cross-reacting with RF borreliae. At present, there are no commercially available serological tests for RF borreliosis in dogs that could improve the diagnosis, i.e., based on GlpQ and BipA [21]. Another RF Borrelia species in Europe, B. miyamotoi, may represent also a diagnostic challenge in terms of cross-reactivity with LB borreliae. Because it has the same vector as B. burgdorferi, co-exposure could be even more probable. Borrelia miyamotoi has already been detected in Ixodes ticks collected from dogs in Germany [22], and was detected in questing Ixodes ticks in Spain [23-25]. Further studies perhaps based on experimental infections may clarify the issue of serological responses related to B. hispanica or B. miyamotoi in dogs.

The cat presented with weight loss, abdominal breathing, and a regenerative anemia, but in contrast to both canine cases, without thrombocytopenia, supporting the idea that the observed reduction in platelets in both dogs could have been the result of a co-infection. As a result of the immediate onset of doxycycline treatment, the culturing of Borrelia in both canine cases was not successful. As the cat blood was taken before the onset of antibiotic treatment, an in vitro cultivation of Borrelia was successful (Figure 1B), but only with an RF-medium (MKP basic medium supplemented with FCS). Highly mobile spirochetes were observed after 10 days of cultivation. In the BSK-H medium, a non-motile spirochete was found, while in the MKP medium and BSK-Y medium, no spirochetes were observed.

In the molecular analyses of $16 \mathrm{~S}$ rRNA and MLST, PCR products were obtained for all three samples. For dog 1 and the cat, sequences for several housekeeping loci were obtained, while for dog 2 , only the $16 \mathrm{~S}$ rRNA locus was successfully amplified. To obtain initial information on the species designation of the isolates, a BLAST search was conducted using $16 \mathrm{~S}$ rRNA sequences. In this search, the highest similarity scores (100\%, Table 3) were received for B. hispanica. For four housekeeping loci, $c l p X, p e p X, p y r G$, and recG, sequences of good quality were obtained for the cat isolate, while for dog 1 , three loci produced good sequence data $(\operatorname{clpX}, p y r G$, and $r p l B)$. A comparison of these sequences with the available data in GenBank showed that the highest BLAST scores (97\% or $98 \%$ ) were in all cases for B. crocidurae, B. duttonii, and B. recurrentis, while the BLAST similarity scores dropped to much lower values for B. persica (Table 3). A search in the MLST database at www.pubmlst.org/borrelia revealed that all of the MLST housekeeping sequences showed closest matches to B. hispanica-clpX was identified to be allele 210 and $r p l B$ allele 244, both representing $B$. hispanica. The closest matches (meaning the sequences were not identical, but differed in some bases) were found for pepX to allele 225 (three differences), for pyrG to allele 232 (seven and one differences in dog 1 and the cat, respectively), and for recG to allele 244 (three differences), further confirming that the isolates belonged to B. hispanica.

The phylogenetic analyses of 16S rRNA and the concatenated sequences of four housekeeping loci from the cat isolate showed that the isolates formed a sister clade to B. hispanica (Figures 2 and 3). 


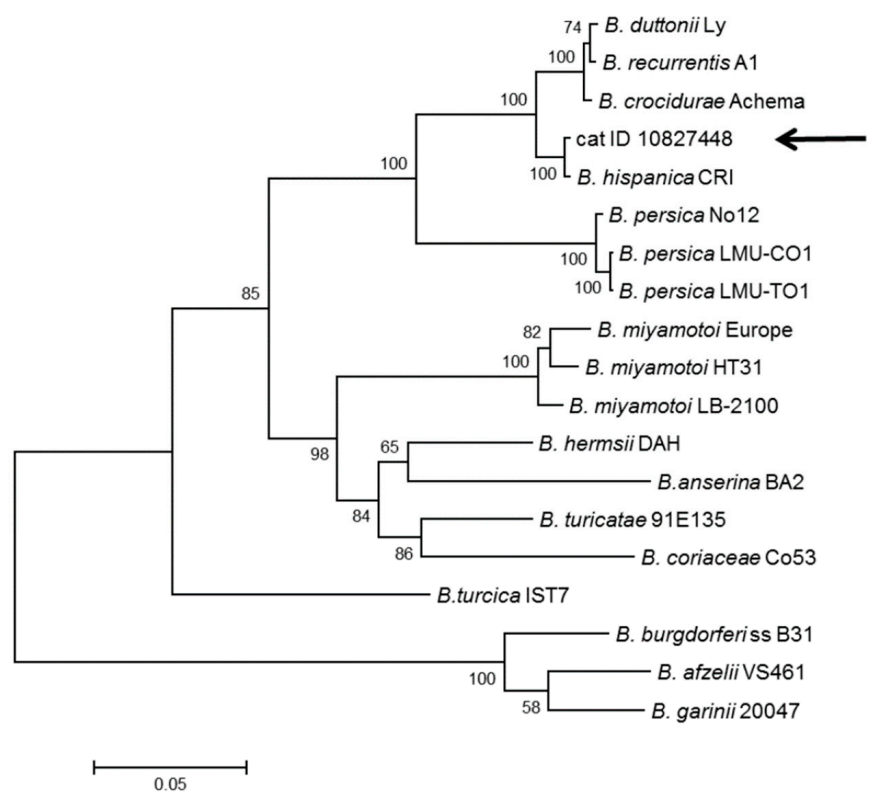

Figure 2. Molecular phylogenetic analysis by the maximum likelihood method based on the sequences of four housekeeping loci ( $\operatorname{lp} X$, pepX, pyrG, and recG). The tree with the highest log likelihood $(-13,651.6897)$ is shown. The bootstrap value (percentage of trees in which the associated taxa clustered together) is shown next to the branches. The tree is drawn to scale; scale bar = number of substitutions per site. The analysis involved 19 nucleotide sequences. There were a total of 2443 positions in the final dataset.

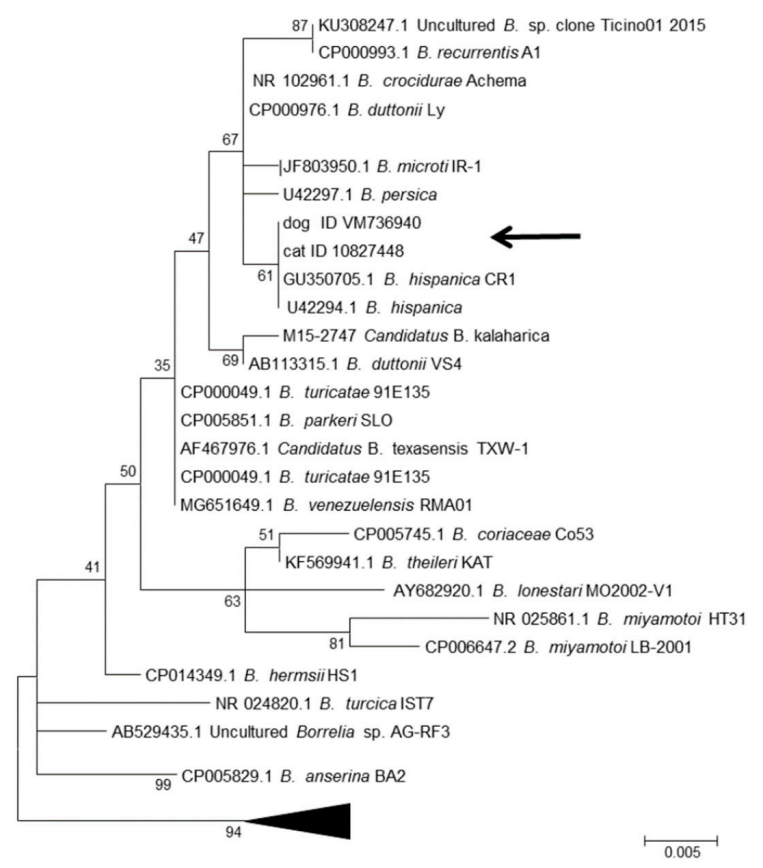

Figure 3. Molecular phylogenetic analysis by maximum likelihood method $16 \mathrm{~S}$ rRNA. The tree with the highest log likelihood (-1091.6698) is shown. The bootstrap value (percentage of trees in which the associated taxa are clustered together) is shown next to the branches. The tree is drawn to scale; scale bar = number of substitutions per site. The analysis involved 43 nucleotide sequences, and the GenBank accession number, species names, and isolate names are given. There were a total of 418 positions in the final dataset. The subtree containing 17 B. burgdorferi sensu lato genospecies is collapsed for clarity. 


\section{Conclusions}

This is the first report of clinical cases caused by the relapsing fever spirochete B. hispanica in dogs and cats from Europe (Spain). Some clinical signs and/or laboratory values might have been influenced by the presence of other vector-borne pathogens.

Author Contributions: N.P., M.G., J.L., J.R., L.H., N.S. and A.C., medical investigation, diagnosis, and treatment of animals, acquisition of samples; G.M. and H.H., molecular analyses and imaging; G.M., N.P. and V.F. wrote the manuscript. All authors have read and agreed to the published version of the manuscript.

Funding: The NRZ Borrelia was funded by the Robert-Koch Institut, Berlin, Germany.

Conflicts of Interest: There is no conflict of interests.

\section{References}

1. Gofton, A.; Margos, G.; Fingerle, V.; Hepner, S.; Loh, S.-M.; Ryan, U.M.; Irwin, P.; Oskam, C.L. Genome-wide analysis of Borrelia turcica and 'Candidatus Borrelia tachyglossi' shows relapsing fever-like genomes with unique genomic links to Lyme disease Borrelia. Infect. Genet. Evol. 2018, 66, 72-81. [CrossRef] [PubMed]

2. Loh, S.-M.; Gillett, A.; Ryan, U.; Irwin, P.; Oskam, C.L. Molecular characterization of 'Candidatus Borrelia tachyglossi' (family Spirochaetaceae) in echidna ticks, Bothriocroton concolor. Int. J. Syst. Evol. Microbiol. 2017, 67, 1075-1080. [CrossRef] [PubMed]

3. Pantchev, N.; Pluta, S.; Huisinga, E.; Nather, S.; Scheufelen, M.; Vrhovec, M.G.; Schweinitz, A.; Hampel, H.; Straubinger, R. Tick-borne Diseases (Borreliosis, Anaplasmosis, Babesiosis) in German and Austrian Dogs: Status quo and Review of Distribution, Transmission, Clinical Findings, Diagnostics and Prophylaxis. Parasitol. Res. 2015, 114, 19-54. [CrossRef] [PubMed]

4. Pantchev, N.; Vrhovec, M.G.; Pluta, S.; Straubinger, R. Seropositivity of Borrelia burgdorferi in a cohort of symptomatic cats from Europe based on a C6-peptide assay with discussion of implications in disease aetiology. Berl. und Munchener Tierarztliche Wochenschr. 2016, 129, 333-339.

5. Talagrand-Reboul, E.; Boyer, P.H.; Bergström, S.; Vial, L.; Boulanger, N. Relapsing Fevers: Neglected Tick-Borne Diseases. Front. Cell. Inf. Microbiol. 2018, 8, 98. [CrossRef]

6. Palma, M.; De Carvalho, I.L.; Figueiredo, M.; Amaro, F.; Boinas, F.; Cutler, S.; Núncio, M.S. Borrelia hispanica in Ornithodoros erraticus, Portugal. Clin. Microbiol. Infect. 2012, 18, 696-701. [CrossRef]

7. Trape, J.-F.; Diatta, G.; Arnathau, C.; Bitam, I.; Sarih, M.; Belghyti, D.; Bouattour, A.; Elguero, E.; Vial, L.; Mané, Y.; et al. The Epidemiology and Geographic Distribution of Relapsing Fever Borreliosis in West and North Africa, with a Review of the Ornithodoros erraticus Complex (Acari: Ixodida). PLoS ONE 2013, 8, e78473. [CrossRef]

8. Baneth, G.; Nachum-Biala, Y.; Halperin, T.; Hershko, Y.; Kleinerman, G.; Anug, Y.; Abdeen, Z.; Lavy, E.; Aroch, I.; Straubinger, R. Borrelia persica infection in dogs and cats: Clinical manifestations, clinicopathological findings and genetic characterization. Parasites Vectors 2016, 9, 244. [CrossRef]

9. Piccione, J.; Levine, G.; Duff, C.; Kuhlman, G.; Scott, K.; Esteve-Gassent, M. Tick-Borne Relapsing Fever in Dogs. J. Vet. Intern. Med. 2016, 30, 1222-1228. [CrossRef]

10. Dandrieux, J.R.; Sacchini, F.; Harms, G.; Globokar, M.; Balzer, H.-J.; Pantchev, N. Canine Leishmania infantum infection: An imported case in UK after staying in the Canary Islands. Parasitol. Res. 2017, 117, 331-334. [CrossRef]

11. Dyachenko, V.; Pantchev, N.; Balzer, H.-J.; Meyersen, A.; Straubinger, R. First case of Anaplasma platys infection in a dog from Croatia. Parasites Vectors 2012, 5, 49. [CrossRef] [PubMed]

12. Preac-Mursic, V.; Wilske, B.; Schierz, G. European Borrelia burgdorferi isolated from humans and ticks culture conditions and antibiotic susceptibility. Zentralbl. Bakteriol. Mikrobiol. Hyg. A 1986, 263, 112-118. [CrossRef]

13. Margos, G.; Stockmeier, S.; Hizo-Teufel, C.; Hepner, S.; Fish, D.; Dautel, H.; Sing, A.; Dzaferovic, E.; Rieger, M.; Jungnick, S.; et al. Long-term in vitro cultivation of Borrelia miyamotoi. Ticks Tick-borne Dis. 2015, 6, 181-184. [CrossRef] [PubMed]

14. Radulović, Ž.; Milutinović, M.; Tomanović, S.; Mulenga, A. Detection of Borrelia-specific $16 \mathrm{~S}$ rRNA sequence in total RNA extracted from Ixodes ricinus ticks. Arq. Bras. Med. Veterinária Zootec. 2010, 62, 862-867. [CrossRef] 
15. Jungnick, S.; Margos, G.; Rieger, M.; Dzaferovic, E.; Bent, S.J.; Overzier, E.; Silaghi, C.; Walder, G.; Wex, F.; Koloczek, J.; et al. Borrelia burgdorferi sensu stricto and Borrelia afzelii: Population structure and differential pathogenicity. Int. J. Med Microbiol. 2015, 305, 673-681. [CrossRef]

16. Nei, M.; Kumar, S. Molecular Evolution and Phylogenetics; Oxford University Press: New York, NY, USA, 2000.

17. Tamura, K.; Peterson, D.; Stecher, G.; Nei, M.; Kumar, S.; Peterson, N. MEGA5: Molecular Evolutionary Genetics Analysis Using Maximum Likelihood, Evolutionary Distance, and Maximum Parsimony Methods. Mol. Biol. Evol. 2011, 28, 2731-2739. [CrossRef]

18. Altschul, S.F.; Gish, W.; Miller, W.; Myers, E.W.; Lipman, D.J. Basic local alignment search tool. J. Mol. Biol. 1990, 215, 403-410. [CrossRef]

19. Kimura, M. A simple method for estimating evolutionary rates of base substitutions through comparative studies of nucleotide sequences. J. Mol. Evol. 1980, 16, 111-120. [CrossRef]

20. Tavaré, S. Some probabilistic and statistical problems in the analysis of DNA sequences. In Some Mathematical Questions in Biology: DNA Sequence Analysis; Miura, R.M., Ed.; American Mathematical Society: Providence, RI, USA, 1986; Volume 17, pp. 57-86.

21. Gettings, J.R.; Lopez, J.E.; Krishnavahjala, A.; Armstrong, B.A.; Thompson, A.T.; Yabsley, M.J. Antibodies to Borrelia turicatae in Experimentally Infected Dogs Cross-React with Borrelia burgdorferi Serologic Assays. J. Clin. Microbiol. 2019, 57. [CrossRef]

22. Schreiber, C.; Krücken, J.; Beck, S.; Maaz, D.; Pachnicke, S.; Krieger, K.; Groß, M.; Kohn, B.; Von Samson-Himmelstjerna, G. Pathogens in ticks collected from dogs in Berlin/Brandenburg, Germany. Parasites Vectors 2014, 7, 535. [CrossRef]

23. Palomar, A.M.; Portillo, A.; Santibanez, P.; Santibanez, S.; Oteo, J.A. Borrelia miyamotoi: Should this pathogen be considered for the diagnosis of tick-borne infectious diseases in Spain? Enferm. Infecc. Microbiol. Clinica 2018, 36, 568-571. [CrossRef]

24. Remesar, S.; Díaz, P.; Venzal, J.M.; Prieto, A.; Estrada-Peña, A.; López, C.M.; Panadero, R.; Fernández, G.; Díez-Baños, P.; Morrondo, P. Longitudinal Study of Infection with Borrelia spp. in Questing Ticks from North-Western Spain. Vector Borne Zoonotic Dis. 2019, 19, 785-792. [CrossRef]

25. Díaz, P.; Arnal, J.L.; Remesar, S.; Pérez-Creo, A.; Venzal, J.M.; Vázquez-López, M.; Prieto, A.; Fernández, P.D.; López, C.M.; Panadero, R.; et al. Molecular identification of Borrelia spirochetes in questing Ixodes ricinus from northwestern Spain. Parasites Vectors 2017, 10, 615. [CrossRef] 